\title{
ZWEI BILDNISKÖPFE DES 2. JHS. N. CHR. VON SAMOS
}

\author{
Özet \\ Samos'tan M.S. 2. yy’a Tarihlenen İki Portre Baş
}

\begin{abstract}
Bu makalede Samos’ta bulunan Roma İmparatorluk Dönemi’ne (M.S. 2. yy) tarihlenen iki mermer portre baş ele alınmıştır. Bu zamana kadar adadaki portre buluntuları içerisinde böyle bir buluntunun olmayışı ve M.S. 2. yüzyıl portrelerinin çok az bir kısmının yayınlanmış olması, bu portre buluntuların önemini artırmaktadır. Bu yüzden ele alınan portreler, Samos için büyük önem taşımaktadır. Bu nedenle bu önemli konuyu Hocam Ord. Prof. Dr. Ekrem Akurgal'ın anısına yayımlamaktayım.
\end{abstract}

Hadrian Dönemi'nde yapımına başlanan hamam kompleksi, M.S. 147/148 depreminden sonra ikinci yapı evresinde tamamlanabilmiştir. Bu yapıdaki onarımlar, M.S. 3. yüzyıl sonuna kadar tespit edilebilmektedir. Yine Hadrian Dönemi'ne tarihlenen Aquadükt ve su yolu, Hadrian Dönemi hamam yapısı ile ilişkilendirilmiştir. Kastro tepesindeki büyük bir alanı kaplayan villa kompleksinin Hadrian Dönemi'ne kadar yoğun olarak kullanılmış olduğu tespit edilmiştir. Heraion'da ise M.S. geç 1. yüzyıldan erken 3. yüzyıla kadar değişiklik ve tamiratlar söz konusudur. Yukarıda adı geçen yapılardan dolayı Samos antik kenti Roma Dönemi’nde özellikle Hadrian Dönemi’nde de önemini korumuş olmalıdır.

Kötü korunmuş olmalarına rağmen her iki portre baş, iyi işçiliğe sahiptir. Dolayısıyla söz konusu bu başlar, bu zamana kadar portre sanatında az araştırılmış olan Samos'un önemini artırmaktadır. Bu konuda Samos'ta özellikle Kastro ikiz tepesinde yapılacak olan sistematik kazılar büyük olasılıkla yeni bilgiler verecektir.

Der größte kaiserzeitliche Baukomplex auf Samos sind die aufwendigen Thermenanlagen, deren Errichtung in hadrianischer Zeit begonnen wurde und die nach einem Erdbeben (wohl 147/148 n. Chr.) in einer 2. Bauphase fertig gestellt worden sind ${ }^{1}$. Weitere Umbauten und Erweiterungen lassen sich bis ins späte 3. Jh. n. Chr. nachweisen ${ }^{2}$. Mit der Anlage der Thermen wird der Bau des Aquäduktes und der römischen Wasserleitung in Verbindung gebracht ${ }^{3}$. Für die großzügige Villenanlage auf dem Kastrohügel von Tigani-Pythagoreion nehmen die Ausgräber eine intensive Nutzung bis in mindestens hadrianische Zeit und eine Zerstörung 177 n. Chr. durch ein Erdbeben oder erst 267 n. Chr. durch die Heruler an ${ }^{4}$. Im Heraion lassen sich

\footnotetext{
Martini $1984233 \mathrm{ff}$.

2 Martini 1984, 250 ff. 261f.

3 Tölle 1969 55; Jantzen 1968,692; Jantzen 1969, 164; Martini 1984, 24. 172.

4 Tölle-Kastenbein 1974, 69.
}

zwar seit dem späteren 1. Jh. n. Chr. Verfalls-erscheinungen feststellen, aber immerhin fallen auch hier aufwendige Baumaßnahmen wie die Pflasterung der heiligen Straße in das späte 2. bzw. frühe 3. Jh. n. Chr. ${ }^{5}$. Zumindest im 2. Jh. n. Chr. war Samos also keineswegs bedeutungslos; vor allem für Hadrian gibt es zahlreiche Inschriften $^{6}$, und es wird erwogen, dass der Kaiser in Zusammenhang mit seiner zweiten Orientreise 129 n. Chr. die Insel besucht haben könnte?

In den Porträtfunden der Insel spiegelt sich ein solcher Befund bisher nicht wider, und von den wenigen Bildnissen des 2. Jhs. n. $\mathrm{Chr}$. ist nur wenig publiziert. Das herausragendste Werk ist die kolossale Statue des

\footnotetext{
5 Kyrieleis 1981, 51f.

6 Herrmann 1960, 123ff.; Tölle-Kastenbein 1974, 69. 175f.; Transier 1985, 131.

7 Weber 1907, 212; Herrmann 1960, 124; Transier 1985, 131.
} 
Trajan aus dem Areal auf dem Kastrohügel ${ }^{8}$. Kein genauerer Fundort auf der Insel ist für einen verriebenen Porträtkopf des Marc Aurel $^{9}$ festgehalten, das Gleiche gilt für eine kopflose Büste mit verziertem Indextäfelchen ${ }^{10}$, die - der Qualität der Arbeit nach zu urteilen - einen sicher sehr guten weiblichen Bildniskopf späthadrianischer bis frühantoninischer Zeit getragen haben muss.

Angesichts dieser spärlichen Befunde kommt den beiden Porträts, die im folgenden in dankbarer Erinnerung an meinen verehrten Lehrer Ekrem Akurgal bekannt gemacht werden sollen, trotz ihres fragmentierten Zustandes durchaus Bedeutung zu.

Bei dem ersten handelt es sich um das Bildnis eines Knaben im Magazin des Museums von Samos $^{11}$. Es ist aus weißem, feinkörnigem Marmor gearbeitet und misst $16,5 \mathrm{~cm}^{12}$. Der Hinterkopf war angestückt ${ }^{13}$; in der Mitte der schrägen, geglätteten Anschlussfläche liegt ein Stiftloch ${ }^{14}$. Ein ausgesplitterter Bruch durchzieht den Kopf schräg von der rechten Wange durch das linke Auge zur linken Schläfe. Die Nase, die rechte Wange, der Mund (bis auf seinen linken Winkel) sowie das Kinn sind fortgebrochen. Der äußere Teil des rechten Auges fehlt, das linke ist verletzt, von den Brauen sind nur die inneren Ansätze und der äußere Teil des linken erhalten. Der Rand des linken Ohres ist beschädigt, vom rechten ist nur der innere Teil erhalten. Von

8 Freyer-Schauenburg 2002, 257ff.

9 Fittschen 1999, 22. 28f. B 2 Taf. 26.

${ }^{10}$ Freyer-Schauenburg 1980, 119 Nr. 11 Taf. 42,1

11 Das Stück ist in dem um 1915 angelegten Inventar von B. Theophanides unter der Nr. 588 verzeichnet; ein genauerer Fundort ist nicht festgehalten. Athen Inst. Neg. 76/858-859.

12 Die Oberfläche ist dicht versintert. Die starke Beschädigung im Gesicht weist ebenso wie die linke Seite des Halsansatzes frische Bruchflächen auf. Weitere Maße: Kinn-Haaransatz 11,5 cm. Augenabstand innen $2,1 \mathrm{~cm}$.

$13 \mathrm{Zu}$ dieser - nicht nur bei Porträtköpfen - öfter belegten Technik (,capita desecta") Crawford 1917, $103 \mathrm{ff}$. Facenna 1955, 25ff. Strocka 1967,132f. 136 Anm. 52. Schauenburg 1967, 55 mit Anm. 74.

${ }^{14}$ Dm. 0,61 cm. T. $1,65 \mathrm{~cm}$. den Haarsträhnen sind nur noch die stark verriebenen Enden vorhanden.

Das Gesicht weist einen oval gerundeten Umriss auf; die Wange ist sehr voll, die Oberfläche des Karnats straff und fest. Die tief liegenden Augen scheinen nicht gebohrt zu sein ${ }^{15}$. Die schmalen, konturierten Oberlider sind (ebenso wie die Brauen) hochgewölbt, die Unterlider sind schwer. Nach Ausweis des erhaltenen Restes war der Mund fest geschlossen. Das Haar war in lange, über der Stirnmitte gegabelte Strähnen gegliedert, die auch am Nacken lockeres Volumen aufweisen.

Der Kopf gehört in die Gruppe von Knabenbildnissen, deren Haartracht an die Frisur des Kaisers Trajan angeglichen worden ist $^{16}$, wobei die Gabelung über der Stirnmitte auf dessen 1 . beziehungsweise 3 . Bildnistypus weist ${ }^{17}$. Die straffe Ober-fläche der vollen Wange und der "kränklich" wirkende Eindruck der tief gebetteten Augen lassen sich unter anderem mit der Knabenbüste Istanbul, Mus. 4989 verbinden, deren Datierung in gallienische Zeit durch J. İnan und E. Rosenbaum ${ }^{18}$ von K. Fittschen revidiert wurde, der einen Ansatz in spättrajanisch-hadrianische Zeit erwägt ${ }^{19}$. Die fehlende oder allenfalls sehr zurückhaltende Bohrung der Augen legt für unseren Kopf eine entsprechende Datierung nahe.

Auch der zweite hier publizierte Kopf, das Bildnis eines jungen Mannes, gehört zu

${ }^{15}$ Wegen der starken Versinterung und der Verletzung der entsprechenden Partien ist eine sparsame Bohrung nicht auszuschließen.

16 Zanker 1982, 309f. Fittschen 1984,199; Fittschen 1988, 303ff.; Goette 1989, 454f.; Raeder 2000, 208ff. Kat. Nr. 78f.

${ }^{17} \mathrm{Zu}$ den Bildnistypen des Trajan zuletzt Fittschen 1997, 816ff. Zur Datierung des 1. Typus vgl. Bergmann 1997, 142, vgl. Freyer-Schauenburg 2002, 266.

${ }^{18}$ İnan - Rosenbaum 1966, 98 Nr. 88 Taf. 53,1. 54.

${ }_{19}$ Fittschen 1973, 58f. zu Nr. 88 (mit weiteren Vergleichsstücken); vgl. auch oben Anm. 16. 
dem alten Skulpturenbestand der Insel (Abb. 3. 4); sein genauerer Fundort ist ebenfalls unbekannt ${ }^{20}$. Er ist aus weißgrauem, mittelkristallinischen Marmor gearbeitet und von etwa lebensgroßem Format ${ }^{21}$. Die Rückseite und die rechte Seite der Kalotte fehlen, die untere Bruchfläche verläuft schräg durch den Hals. Alle hervorstehenden Teile des Gesichtes - Brauen, Nase, Mund und Kinn - sind abgesplittert, Augen und Wangen sind bestoßen. Von den Ohren ist nur noch der innere Streifen des linken erhalten. Die Locken sind beschädigt, die Strähne über dem linken inneren Augenwinkel abgesplittert.

Der Kopf war leicht zu seiner Linken gedreht. Ein gleichmäßig gerundeter Umriss umschreibt die vollen Wangen und das Kinn, das nicht abgesetzt ist. Das Orbital hängt in einer weichen Falte über. Die schmalen Oberlider sind scharf konturiert. Am linken Auge hat sich direkt darunter die eingetiefte Pupille noch erhalten, der geritzte Umriss der Iris ist beiderseits noch zu erahnen. Die Unterlider gehen in weiche Tränensäcke über. Von dem fest geschlossenen Mund sind die eingetiefte Mundspalte und die Grübchen an den Mundwinkeln noch erkennbar.

Bisher ist der Kopf in der Literatur nur einmal erwähnt worden - als Bildnis des jugendlichen Caracalla $^{22}$. Mit dem Frisurenschema dieses Prinzen $^{23}$ lässt sich die Anordnung der Stirnlocken unseres Kopfes jedoch nicht verbinden. Sie gabeln sich über dem rechten inneren Augen-winkel; über dem inneren Teil des linken Auges lag eine Zange, deren äußerer Schenkel abgesplittert ist. Die anschließende

${ }^{20}$ Der Kopf lag zunächst in einer Vitrine im Museumsraum in Tigani-Pythagoreion, gelangte dann vorübergehend in das Magazin bei den Thermen und hat jetzt im Skulpturenmagazin auf dem Kastrohügel seinen Platz gefunden. Athen Inst. Neg. Samos 2554. Ph. Hubert 387,8-10. 388,11-12. 390,1.

${ }^{21}$ H. $29,5 \mathrm{~cm}$. Kinn-Scheitel $24 \mathrm{~cm}$. Augenabstand innen 3,1 cm, außen 9,6 cm. Br. des Mundes 4,3 cm.

${ }^{22}$ Vermeule 1968, $400 \mathrm{Nr} .3$.

23 Wiggers - Wegner 1971, 17ff.; Fittschen-Zanker 1985, 99f. zu Nr. 86 (F.).
Partie bis zur linken Stirnecke nehmen drei Bündel gleich gerichteter Sichellocken ein, deren Spitzen nach innen weisen; zwei Spitzen spiegelbildlich angeordneter Locken sind über dem äußeren Teil des rechten Auges gerade noch erhalten. Die Strähnen des Schläfenhaares links sind nach unten gewölbt, ihre Spitzen weisen nach hinten. Darunter hängt eine Sichellocke mit nach vorn gerichteter Spitze vor dem Ohr herab, das zumindest im erhaltenen vorderen Teil frei war.

Die Gliederung des Haares, das plastische Volumen der sich teilweise überlagernden Sichellocken sowie physiognomische Merkmale wie die vollen Wangen und die Form der Augen weisen unser Jünglingsbildnis in antoninische Zeit. Es lehnt sich wie u.a. eine Knabenbüste in Farnborough Hall $^{24}$ - an die damaligen Prinzenbildnisse $a^{25}$. In diesen Umkreis passt auch die zurückhaltende Augenbohrung ${ }^{26}$. Eine ähnliche, aus kräftigen Sichellocken bestehende Frisur findet sich bei antoninischen Köpfen in Bordeaux ${ }^{27}$ und Athen, N.M. 556 ${ }^{28}$, wobei die Strähnen bei letzterem allerdings Bohrgänge aufweisen. Die teilweise Hinterarbeitung der plastischen Stirnlocken mit den zum Teil tiefen Zwischenräumen verbinden das samische Jünglingsbildnis mit

${ }^{24}$ Scholl 1995, 58f. F 17 Taf. 39,3/4. 40.

${ }^{25}$ Fittschen 1999, 79 mit Anm. 411 Taf. 129 a.b.d) vergleicht die Büste mit den Bildnistypen C (Fittschen 1999, 32 ff. Taf. 58 ff.: Knabenbildnis des Lucius Verus) und G (Fittschen 1999, 50 ff. Taf. 78 f.: Knabenbildnis des Galerius). Unser Kopf steht in Teilen der Stirnhaargliederung und (trotz der etwas höheren Altersstufe) in einigen physiognomischen Zügen wie der Bildung der schmalen Augen mit dem überhängenden Orbital dem Typus $\mathrm{C}$ nahe.

${ }^{26} \mathrm{Ob}$ die Brauen unseres Kopfes schraffiert waren, läßt sich nicht mehr feststellen, da diese zu Gänze abgesplittert sind.

${ }^{27}$ Fittschen 1999, 89 Nr. 64 Taf. 159b.

28 Datsulis-Stavridis 1982, 217f. Taf. 51b. Fittschen 1999, 89 Nr. 65. 
einem qualitätvollen Porträt im Kerameikosmuseum, das von H. Riemann in das 3. Viertel des 2. Jhs. $n$. Chr. datiert worden ist ${ }^{29}$.

Die beiden Bildnisköpfe, die hier vorgelegt worden sind, lassen trotz der starken Beschädigungen noch erkennen, dass es sich um gute Arbeiten gehandelt hat. Sie tragen dazu bei, das auf dem Gebiet der Porträtkunst bisher sehr unvollständige Bild von Samos im 2. Jh. n. Chr. zu bereichern. Weitere Erkenntnisse (nicht nur auf diesem Feld) wären von systematischen Grabungen im Stadtgebiet, vor allem auf dem Doppelhügel des Kastro, mit großer Wahrscheinlichkeit zu erwarten.

Dr. Brigitte Freyer-Schauenburg

Archäologisches Institut

Christian-Albrecht-Universität

Olshausenstr. 40-50

24118 Kiel - Deutschland

${ }^{29}$ Riemann 1940, 89f. Nr. 119 Taf. 27. 


\section{Abbildungs-Verzeichnis}

Abb. 1. 2 Knabenbildnis in Samos, Mus. Magazin Inv. 588.

Abb. 3. 4 Bildnis eines jungen Mannes in Pythagoreion, Kastro Magazin.

\section{Abbildungs-Nachweise}

Ab. 1. 2 Athen Inst.Neg.

Abb. 3. 4 Ph. Hubert (im Samosarchiv im DAI Athen). 


\section{Bibliographie}

Bergmann 1997

Crawford 1917

Datsulis-Stavridis 1982

Facenna 1955

Fittschen 1984

Fittschen 1988

Fittschen 1997

Fittschen 1999

Fittschen - Zanker 1983

Fittschen - Zanker 1985

Freyer-Schauenburg 1980

Freyer-Schauenburg

2002

Goette 1989

Herrmann 1960

İnan - Rosenbaum 1966

Jantzen 1968

Jantzen 1969

Kyrieleis 1981

Martini 1984

Raeder 2000

Riemann 1940

Schauenburg 1967

Scholl 1995

Strocka 1967

Tölle 1969

Tölle-Kastenbein 1974

Transier 1985

Vermeule 1968

Weber 1907

Wiggers - Wegner 1971

Zanker 1982
M. Bergmann, Zu den Porträts des Trajan und Hadrian, in: Italica $M M C C$. Actas de las jornadas del 2200 aniversario de la fondacion de Italica, Sevilla 1994 (1997, Hrsg. A. Caballos - P. Leon) 142-153.

J. Crawford, Capita desecta and marble coiffures, MemAmAcc 1, 1917, 103119.

A. Datsulis-Stavridis, Portraita tis epochis ton Antoninon sto Ethniko Archaiologiko Mousieo Athinon, AEphem 1982, 215-226.

D. Facenna, Ritratto di giovane donna da Bagni di Tivoli, ArchCl 7, 1955, 24-32.

K. Fittschen, Rez. Zu J. İnan - E. Alföldi-Rosenbaum, Römische und frühbyzantinische Porträtplastik aus der Türkei. Neu Funde, GGA 236, 1984, 188210.

K. Fittschen, Kinderporträt und offizielles Porträt im 2. Jh. n. Chr., in: Ritratto ufficiale e ritratto privato (1988) 303-306.

K. Fittschen, Traiano, in: EAA Suppl. V (1997) 816-818.

K. Fittschen, Prinzenbildnisse antoninischer Zeit (1999).

K. Fittschen - P. Zanker, Katalog der römischen Porträts in den Capitolinischen Museen und den anderen kommunalen Sammlungen der Stadt Rom. Band III. Kaiserrinnen - und Prinzessinnen Bildnisse, Frauenporträts (1983).

K. Fittschen - P. Zanker, Band I. Kaiser- und Prinzenbildnisse (1985).

B. Freyer-Schauenburg, Büsten mit reliefverzierten Indextäfelchen, in: Eikones. Festschrift H. Jucker (1980) 118-125.

B. Freyer-Schauenburg, Die Statue des Trajan auf Samos, $A M 117,2002$, 257-295.

Goette, Beobachtungen zu römischen Kinderportraits, AA 1989, 453-471.

P. Herrmann, Inschriften römischer Zeit aus dem Heraion von Samos, $A M$ 75, 1960, 68-183.

J. İnan - A. Rosenbaum, Roman and early byzantine portrait sculpture in Asia Minor (1966).

U. Jantzen, Samos 1967, AA 1968, 687-692.

U. Jantzen, Samos 1968, AA 1969, 161-165.

H. Kyrieleis, Führer durch das Heraion von Samos (1981).

W. Martini, Samos XVI. Das Gymnasium von Samos (1984).

J. Raeder, Die antiken Skulpturen in Petworth House (2000).

H. Riemann, Kerameikos II. Die Skulpturen (1940).

K. Schauenburg, Perückenträgerin im Blattkelch, StädelJb 1967, 45-63.

A. Scholl, Die antiken Skulpturen in Farnborough Hall (1995).

V. M. Strocka, Aphroditekopf in Brescia, JdI 82, 1967, 110-156.

R. Tölle, Die antike Stadt Samos (1969).

R. Tölle-Kastenbein, Samos XIV. Das Kastro Tigani (1974).

W. Transier, Samiaka (1985).

C. Vermeule, Roman Imperial Art in Greece and Asia Minor (1968).

W. Weber, Untersuchungen zur Geschichte des Kaisers Hadrian (1907).

W. Wiggers - M. Wegner, Caracalla, Geta, Plautilla, Macrinus bis Balbinus. Das Römische Herrscherbild III, 1 (1971).

P. Zanker, Herrscherbild und Zeitgesicht, in: Wiss. Zeitschr. der HumboldtUniv. 2/3, 1982, 307-312. 


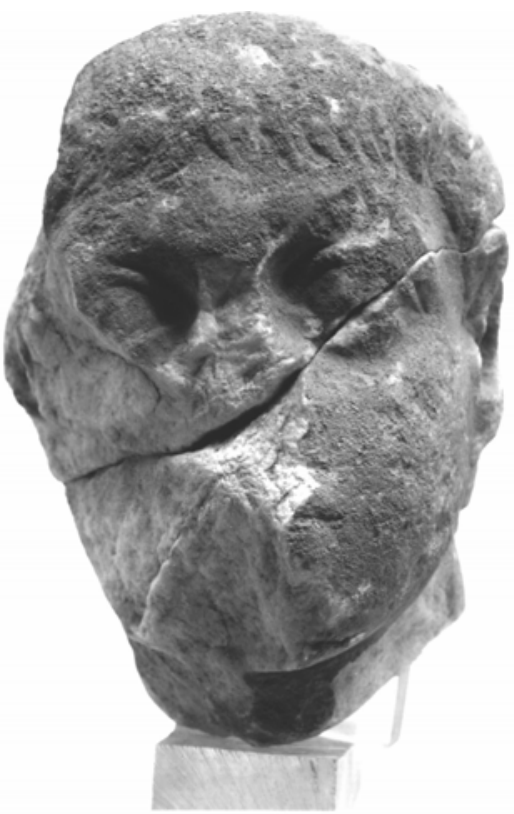

Abb.1

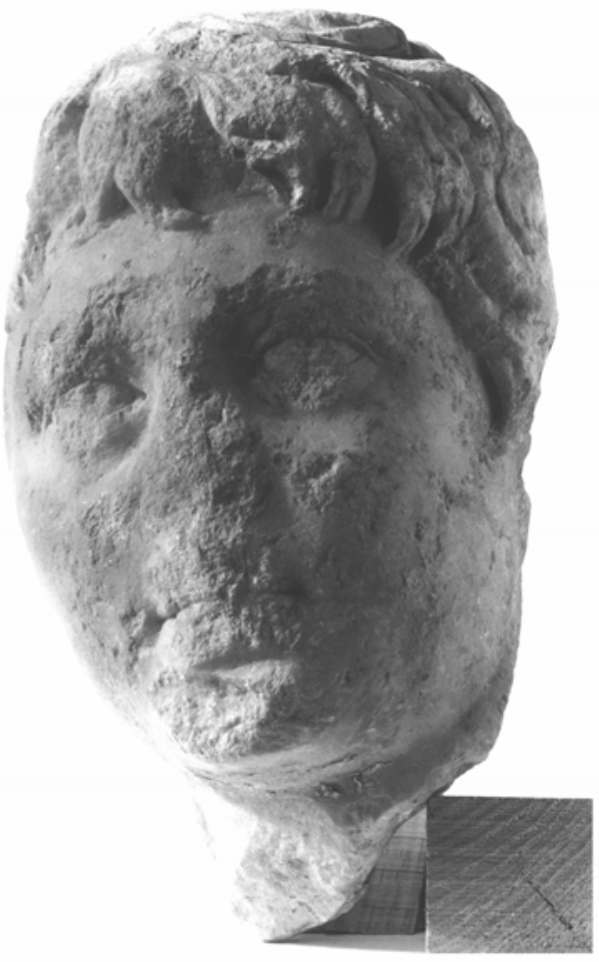

Abb. 3

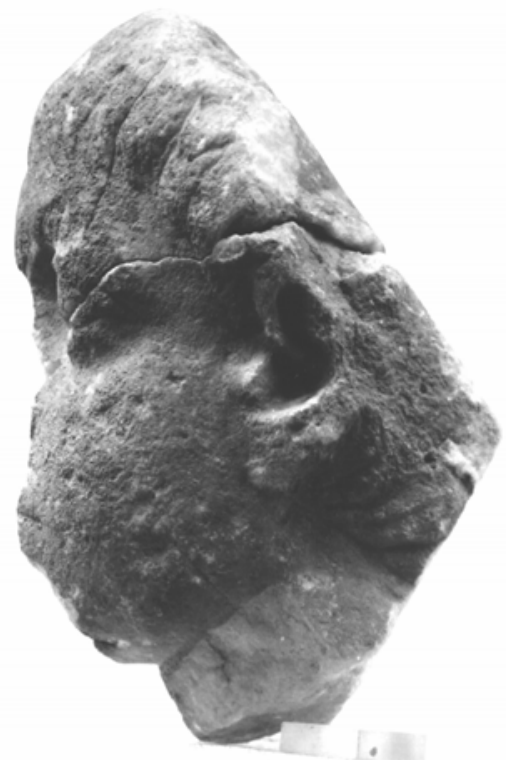

Abb. 2

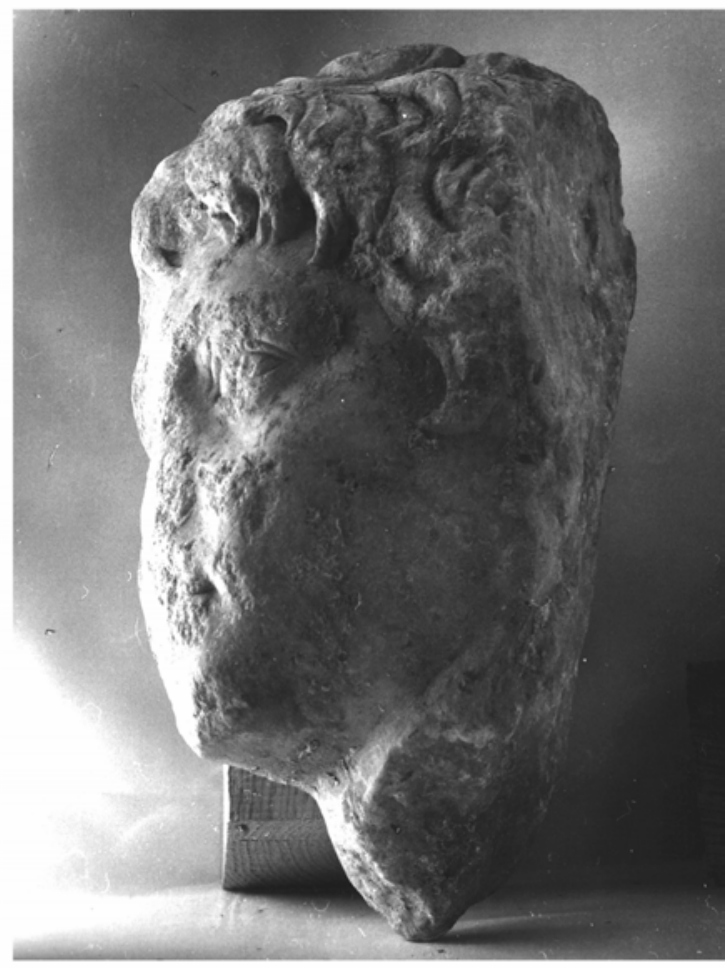

Abb. 4 